adversely affect the course of IBD. The authors hypothesise that: (1) anxiety and stress are more common in acute severe ulcerative colitis (ASUC) than in patients with inactive UC; (2) anxiety and stress are more common in patients presenting for the first time with UC; (3) anxiety and stress worsen outcome in ASUC.

Methods 39 ASUC patients (aged 28 (18-74) years (median (range)); disease duration $10(0-240)$ months), requiring hospital admission and intravenous hydrocortisone with a Baron's sigmoidoscopic score $\geq 2$, completed questionnaires to assess anxiety (Hospital Anxiety and Depression Score (HADS-A)) and stress levels (Perceived Stress Questionnaire: Recent, R-PSQ, General, G PSQ). Outcomes were assessed, using the Travis criteria (day 3 ) and colectomy rates at day 70 , in relation to upper and lower tertiles of the anxiety and stress scores. In addition, the psychometric scores of the ASUC patients were compared to those of 27 outpatients with inactive UC.

Results Patients with ASUC had higher stress levels (R-PSO Index $0.52(0.03)$ (mean (SEM)), G-PSO $0.46(0.03)$ ) than in patients with inactive UC $(0.35(0.03), p=0.001,0.39(0.02)$, $\mathrm{p}=0.046$, respectively) but there was no difference in anxiety scores (HADS-A 9.6 (0.8), 8.4 (0.7), $\mathrm{p}=0.9$, respectively). However, in patients with ASUC, there was a positive correlation between stress and anxiety scores (R-PSQ, $r 2=0.27$, $\mathrm{p}=0.0008$; G-PSQ, $\mathrm{r} 2=0.14, \mathrm{p}=0.02)$ ). Although stress scores were similar, anxiety levels were higher in the 11 newly presenting ASUC patients (12 (1.4)) than in those previously diagnosed (8.6 (0.9), $p=0.049)$. There was no difference in those responding to intravenous hydrocortisone (by Travis criteria) or in day 70 colectomy rates between patients who were highly (upper tertile) anxious (Travis response 8/13, colectomy rate $2 / 13$ ) or highly (upper tertile) stressed (R-PSO: Travis response 8/12, colectomy rate 6/13; G-PSQ Travis response 5/13, colectomy rate $4 / 13$ ) compared to those less (lower tertile) anxious $(5 / 13,3 / 13$, respectively) or stressed (lower tertile) (R-PSO, 5/13, 1/13; G-PSO 5/12, 1/13, respectively).

Conclusion Perceived stress levels are greater in patients with ASUC than in those in remission and anxiety scores are highest in patients newly presenting with UC, but neither stress nor anxiety influenced disease outcome.

Competing interests None.

Keywords psychological stress, ulcerative colitis.

\section{PTH-067 ANXIETY AND PSYCHOLOGICAL STRESS IN ACUTE SEVERE ULCERATIVE COLITIS: PREVALENCE AND EFFECT ON OUTCOME}

doi:10.1136/gut.2011.239301.468

M Wahed, ${ }^{1, *}$ J R Goodhand, ${ }^{1}$ L Langmead, ${ }^{1}$ P M Irving, ${ }^{2}$ J Sanderson, ${ }^{2}$ S Bloom, ${ }^{3}$ S McCartney, ${ }^{3} \mathrm{~J}$ Mawdsley, ${ }^{4}$ D S Rampton' ${ }^{1}$ Digestives Disorders Clinical Academic Unit, Barts \& The London School of Medicine \& Dentistry, London, UK; ${ }^{2}$ Department of Gastroenterology, Guy's \& St Thomas' Hospitals NHS Foundation Trust, London, UK; ${ }^{3}$ Department of Gastroenterology, University College London Hospitals NHS Foundation Trust, London, UK; ${ }^{4}$ Department of Gastroenterology, West Middlesex University Hospital NHS Trust, London, UK

Introduction There is increasing evidence that psychological stress and associated mood disorders are linked with, and 